\title{
PROPOSTA METODOLÓGICA PARA O AJUSTE ÓTIMO DA DISTRIBUIÇÃO DIAMÉTRICA WEIBULL 3P
}

\author{
Oscar Santiago Vallejos Barra ${ }^{1}$ \\ Carlos Roberto Sanquetta ${ }^{2}$ \\ Julio Eduardo Arce ${ }^{3}$ \\ Sebastião do Amaral Machado ${ }^{4}$ \\ Ana Paula Dalla Corte ${ }^{5}$
}

\begin{abstract}
RESUMO
A distribuição de Weibull de três parâmetros tem ampla utilização na área florestal. Existem três métodos para ajustar a distribuição, os quais consideram o parâmetro de locação como um termo independente que deve ser conhecido para obter os restantes parâmetros. Esta proposta metodológica visa, através de um processo interativo, otimizar o ajuste de cada um dos métodos mais utilizados para esta finalidade, sendo eles: máxima verossimilhança, momentos e percentis. Esta proposta visa minimizar o "dn" do teste de aderência de Kolmogorov-Smirnov. Observou-se que os valores de "dn" da distribuição Weibull de três parâmetros são inferiores aos obtidos na de dois parâmetros nos três métodos de ajuste. Observou-se ainda que os valores "dn" de cada método não apresentam diferenças expressivas, mas quando são comparadas as probabilidades associadas à magnitude tornam-se relevantes e justificam a metodologia proposta. Concluiu-se que esta nova metodologia é uma alternativa útil para ajuste de distribuições diamétricas e aplicações em modelagem do crescimento e da produção de povoamentos florestais.

Palavras-chaves: distribuição, otimização, método de ajuste
\end{abstract}

\section{A NEW METHOD FOR THE OPTIMUM FITTING OF THE 3-P WEIBULL DIAMETER DISTRIBUTION}

\begin{abstract}
The Weibull probability distribution of three parameters has wide use forestry. There are three fitting methods used for this purpose, which take into consideration the location parameter as an independent term that should be known previously to obtain the remaining parameters. This methodological proposal aims at showing an iterative method of optimization the fitting of the three parameters of the Weibull function in each one of the methods: maximum likelihood, moments, and percentiles. The proposed method minimizes the statistical "dn" of the Kolmogorov-Smirnov test of goodness-of-fit. It was noticed that "dn" of the three parameters Weibull distribution are lower than those of the two parameters function for the three fitting methods. It was also observed that "dn" values of each method were not significantly different one another, but when the probabilities were compared expressive differences were noticed, indicating the methodology is adequate. It was concluded that the new methodology is a useful alternative for the fitting of diameter distributions and application in modeling of growth and yield of forest stands.
\end{abstract}

Keywords: distribution, optimization, fitting method

\section{INTRODUÇÃO}

As funções de densidade de probabilidade permitem estimar a provável distribuição que apresentam os diâmetros, descrevendo a estrutura de um povoamento, o qual facilita o planejamento da produção da floresta.

Uma das funções de densidade de probabilidade mais destacadas é a distribuição Weibull de três parâmetros (Expressão [1] ), onde os parâmetros (a) , (b) e (c) são responsáveis pela locação, escala e forma da distribuição respectivamente.

\footnotetext{
${ }^{1}$ Doutorando Manejo Florestal, Escola de Engenharia Florestal, UFPR. E-mail: ovallejo@floresta.ufpr.br

${ }^{2}$ Dr. Professor da área de Manejo Florestal, Escola de Engenharia Florestal, UFPR. E-mail: sanqueta@floresta.ufpr.br

${ }^{3}$ Dr. Professor da área de Manejo Florestal, Escola de Engenharia Florestal, UFPR. E-mail: jarce@floresta.ufpr.br

${ }^{4}$ Dr. Professor da área de Manejo Florestal, Escola de Engenharia Florestal, UFPR, E-mail: sammac@floresta.ufpr.br

${ }^{5}$ Mestranda Manejo Florestal, Prof ${ }^{a}$. da área de Manejo Florestal, Escola de Engenharia Florestal, UFPR, E-mail: anacorte@floresta.ufpr.br

Recebido para publicação: 01/09/2004

Aceito para publicação: 17/12/2004
} 
$f($ Dap $)=\left(\frac{c}{b}\right)\left(\frac{D a p-a}{b}\right)^{c-1} \exp ^{\left(-\left(\frac{D a p-a}{b}\right)^{c}\right)}$

Onde

$$
\begin{aligned}
& 0 \leq a<\text { Dap mínimo } \\
& \text { Dap }>a \\
& b>0 \\
& c>0
\end{aligned}
$$

Para obter os parâmetros da distribuição três métodos de ajuste tem sido descritos na literatura: máxima verossimilhança, momentos e percentis, descritos por inúmeros autores, como por exemplo: o método da máxima verossimilhança foi utilizado por Bailey e Dell (1973), Campos e Turnbull (1981), Finger (1982), Gadow (1983), Glade (1986) e Tiersch (1997). O método dos momentos, empregado por Burk e Newberry (1984), Scolforo (1990), Tiersch (1997), Abreu (2000) e Maestri (2003). O método dos percentis, utilizado por Bailey e Dell (1973), Cao e Burkhart (1984), Bordes et al. (1987), Silva e Bailey (1987), Maestri (1992), Tiersch (1997), Kangas e Maltamo (2000) e Maltamo et al. (2000).

O parâmetro de locação (a) nos três métodos de ajuste apresenta-se como um termo independente que deve ser conhecido previamente para obter o parâmetro de escala $\mathrm{e}$ forma. Na maioria dos casos, através de um processo iterativo, calcula-se o parâmetro (a) como uma percentagem do diâmetro mínimo de cada unidade de amostragem considerada, ou pressupõe-se um certo valor. Posteriormente, obtêm-se os restantes parâmetros e avalia-se a qualidade do ajuste através de testes de aderência (vide Maestri, 2003; Eisfeld, 2004)

$\mathrm{Na}$ maioria dos casos citados foi empregado $\mathrm{o}$ teste de aderência não paramétrico Kolmogorov-Smirnov indicado para avaliar a distribuição teórica de um conjunto de dados (Gadow, 1983; Gibbons e Chakraborti, 1992), já que é muito sensível a qualquer diferença do valor central e da dispersão (Lebart; Morineau; Fénelon, 1985). Este teste baseia-se na máxima diferença entre a distribuição acumulativa dos erros considerando a estatística “dn" (Expressão [2]).

$$
\begin{aligned}
& d_{n}^{+}=\operatorname{Max}\left\{\frac{i}{n}-F\left(\operatorname{Dap}_{i}\right)\right\} \quad d_{n}^{-}=\operatorname{Max}\left\{F\left(\operatorname{Dap}_{i}\right)-\frac{i}{n}+\frac{1}{n}\right\} \mathrm{i}=1,2, \ldots, \mathrm{n} \\
& d_{n}=\operatorname{Max}\left\{d_{n}^{+} ; d_{n}^{-}\right\}
\end{aligned}
$$

A distribuição acumulativa dos dados é obtida ao integrar a expressão [1], cujo resultado apresenta-se na expressão [3].

$$
F(\text { Dap })=1-\exp ^{\left(-\left(\frac{\text { Dap }-a}{b}\right)^{c}\right)}
$$

Dada a relevância da distribuição Weibull na área florestal e considerando as possíveis dificuldades para seu ajuste, o objetivo do presente trabalho é apresentar através de processo interativo de otimização, uma metodologia que otimiza o ajuste da distribuição (a).

\section{PROPOSTA METODOLÓGICA}

A proposta metodológica busca minimizar o valor do estatístico "dn" do teste de Kolmogorov-Smirnov, em cada unidade de amostra analisada, considerando as características de cada método de ajuste. Deste modo pode-se formular um problema de 
otimização para encontrar a solução ótima do ajuste da distribuição Weibull de três parâmetros. Os dados referem-se à distribuição de diâmetros (DAP) de um povoamento florestal, conforme citado em Barra (2003).

A função objetivo descreve-se na expressão [4], e as restrições dos parâmetros na expressão [5]. Os parâmetros de escala e forma são calculados de acordo ao método de ajuste selecionado: método da máxima verossimilhança (expressão [6]), método dos momentos (expressão [7]) e método dos percentis (expressão [8]).

$$
\begin{array}{ccc}
\operatorname{Minimizar}_{n}=\operatorname{Max}\left\{\operatorname{Max}\left\{\frac{i}{n}-F\left(\operatorname{Dap}_{i}\right)\right\} ;\right. & \left.\operatorname{Max}\left\{F\left(\operatorname{Dap}_{i}\right)-\frac{i}{n}+\frac{1}{n}\right\}\right\} \\
\mathrm{a} & > & 0 \\
\mathrm{a} & > & \text { Dap mínimo } \\
\mathrm{c} & 0 \\
n=\frac{\sum_{\mathrm{i}=1}^{\mathrm{n}}\left(\operatorname{Dap}_{\mathrm{i}}-\mathrm{a}\right)^{\mathrm{c}} \operatorname{Ln}\left(\operatorname{Dap}_{\mathrm{i}}-a\right)}{\sum_{\mathrm{i}=1}^{\mathrm{n}}\left(\operatorname{Dap}_{\mathrm{i}}-\mathrm{a}\right)^{\mathrm{c}}}-\sum_{\mathrm{i}=1}^{\mathrm{n}} \operatorname{Ln}\left(\operatorname{Dap}_{\mathrm{i}}-a\right) & ; b=\frac{\left(\sum_{i=1}^{n}\left(\operatorname{Dap}_{\mathrm{i}}-\mathrm{a}\right)^{\mathrm{c}}\right)^{\frac{1}{c}}}{n^{\frac{1}{c}}}
\end{array}
$$

Onde:

$$
\begin{aligned}
& \mathrm{n}=\text { quantidade de observações } \\
& \mathrm{Ln}=\text { Logaritmo natural } \\
& \frac{\sigma_{D a p-a}}{\overline{D a p}-a}=\frac{\sqrt{\Gamma\left(1+\frac{2}{c}\right)-\Gamma^{2}\left(1+\frac{1}{c}\right)}}{\Gamma\left(1+\frac{1}{c}\right)} \quad ; b=\frac{\overline{D a p}-a}{\Gamma\left(1+\frac{1}{c}\right)}
\end{aligned}
$$

Onde:

$$
\sigma_{\text {Dap-a }}=\quad \text { desvio padrão do diâmetro }
$$

$\overline{D a p}-\mathrm{a}=\quad$ diâmetro médio aritmético

$$
\Gamma \quad=\quad \text { função Gamma }
$$

$c=\frac{\sum_{i=1}^{p} x_{i}^{2}-\frac{\left(\sum_{i=1}^{p} x_{i}\right)^{2}}{n}}{\sum_{i=1}^{p} x_{i} y_{i}-\frac{\sum_{i=1}^{p} x_{i} \sum_{i=1}^{p} y_{i}}{n}}$

Onde:

$$
\begin{array}{lll}
\mathrm{y}_{\mathrm{i}} & = & \operatorname{Ln}\left(\text { Percentil }_{\mathrm{i}}(\text { Dap }-\mathrm{a})\right) \\
\mathrm{x}_{\mathrm{i}} & = & \operatorname{Ln}(-\operatorname{Ln}(1-\text { Percentil } \\
\mathrm{p} & = & \text { quantidade de percentis utilizados }
\end{array}
$$




\section{IMPLEMENTAÇÃO E APLICAÇÃO NUMÉRICA}

Com o objetivo de testar a metodologia proposta, utilizou-se o aplicativo de otimização não linear "Solver.xla" do Microsoft Excel 2000®, definindo a função objetivo e restrições da cada método de ajuste.

A informação requerida para a aplicação numérica foi obtida dos dados de diâmetro e altura do peito (DAP) de povoamento florestal do híbrido Populus $\mathrm{x}$ euramericana utilizados por Barra (2003).

Ajustou-se a distribuição de Weibull de três parâmetros, através da metodologia proposta e a mesma distribuição, mas considerando o parâmetro de locação com um valor zero (Distribuição Weibull de dois parâmetros) a fim de ter um ponto de referência para a comprovação da minimização da estatística "dn".

$\mathrm{Na}$ tabela 1 apresenta-se o resultado do ajuste da distribuição Weibull de dois e três parâmetros, onde é possível observar que os valores "dn" da distribuição Weibull de três parâmetros são inferiores aos obtidos na distribuição de dois parâmetros nos três métodos de ajuste. Ao comparar os valores "dn" de cada método as diferenças não parecem expressivas, mas quando são comparadas as probabilidades associadas à magnitude torna-se relevante e justifica a metodologia proposta.

Neste caso a distribuição de Weibull de três parâmetros foi mais acurada que a distribuição de dois parâmetros, no entanto, em algum caso particular é possível que o resultado da otimização indique que o valor do parâmetro de locação (a) seja zero.

Tabela 1: Resultado da estimação da distribuição de Weibull de dois e três parâmetros segundo método de ajuste

Table 1: Estimation of weibull distribution for the two and three parameter functions

\begin{tabular}{rrrrrrr}
\hline & \multicolumn{2}{r}{ Método da máxima verossimilhança } & Método dos momentos & \multicolumn{2}{r}{ Método dos percentis } \\
\cline { 2 - 7 } & Weibull 2P & Weibull 3P & Weibull 2P & Weibull 3P & Weibull 2P & Weibull 3P \\
\hline Parâmetro "a" & 0,000 & 15,135 & 0,000 & 15,500 & 0,000 & 14,850 \\
Parâmetro "b" & 27,445 & 5,199 & 27,408 & 5,054 & 27,363 & 5,312 \\
Parâmetro "c" & 12,018 & 12,171 & 13,051 & 11,804 & 12,484 & 12,460 \\
"dn" & 0,043 & 0,020 & 0,048 & 0,021 & 0,035 & 0,020 \\
Probabilidade & 0,387 & 0,810 & 0,305 & 0,803 & 0,532 & 0,806 \\
\hline
\end{tabular}


Na Figura 1, apresenta-se graficamente o resultado do ajuste da distribuição Weibull de dois e três parâmetros, segundo o método de ajuste. Em termos gerais observa-se que os métodos não diferem notoriamente entre si.
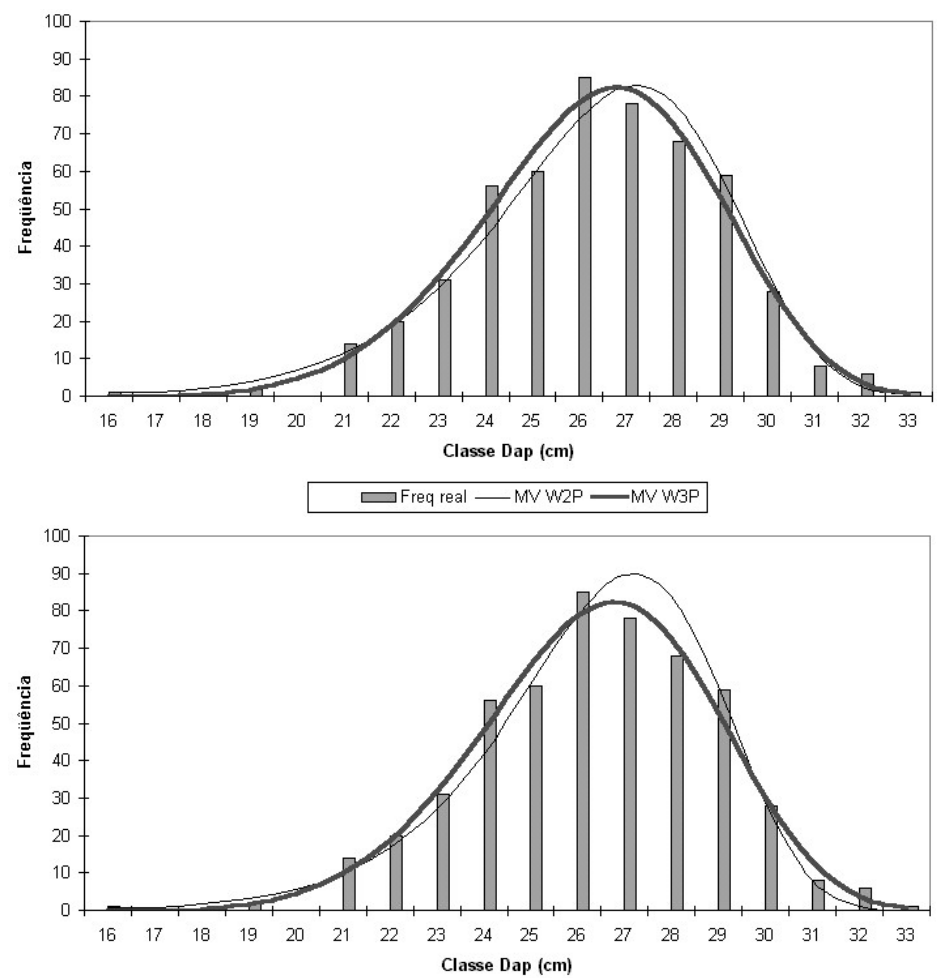

a)

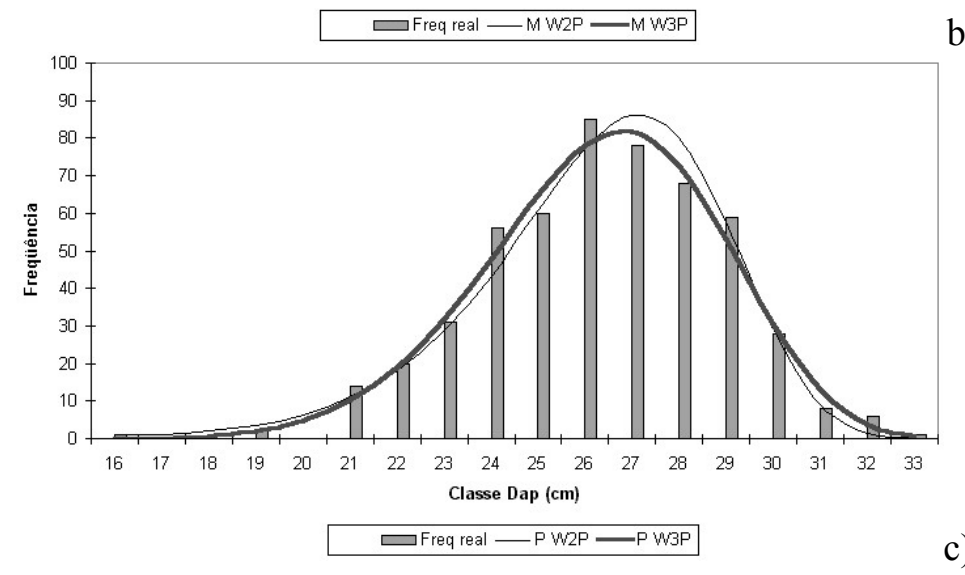

b)

c)

Figura 1: Representação gráfica do ajuste da distribuição Weibull de dois e três parâmetros: a) método da máxima verossimilhança, b) método dos momentos e c) método dos percentis

Figure 1: Representation of the adjustment of the Weibull distribution for the two and three parameter functions: a) maximum likelihood method, b) moments method, c) percentiles method 


\section{CONCLUSÕES E RECOMENDAÇÕES}

As conclusões e recomendações deste trabalho são as seguintes:

1. A metodologia proposta é uma alternativa de ajuste da distribuição Weibull de três parâmetros, possibilitando o ajuste ótimo da distribuição;

2. No caso específico dos dados utilizados na exemplificação da metodologia proposta não houve diferença significativa entre os resultados de ajuste utilizando as funções de dois e três parâmetros no tocante aos valores "dn" do teste de Kolmogorov e Smirnov. Todavia, quando comparadas as probabilidades associadas a cada caso, verifica-se diferenças expressivas, justificando o método proposto;

3. A metodologia utilizada não requer o uso de pacotes estatísticos e métodos tentativos, que podem implicar em erros na otimização global do ajuste e possível indecisão pelo usuário;

4. Recomenda-se o uso desta metodologia para ajuste de distribuições diamétricas e para a construção de modelos de crescimento e produção de povoamentos florestais em geral.

\section{REFERÊNCIAS}

ABREU, E. Modelagem da prognose precoce do volume por classe diamétrica para Eucalyptus grandis. Lavras, 2000, 70 f. Tese (Mestrado em Manejo Florestal) Universidade Federal de Lavras.

BAILEY, R.; DELL, T. Quantifying diameter distributions with the Weibull Function. Forest Sci. v. 19, n 2. p. 97 - 104. 1973

BARRA, O. Sistema de simulação de crescimento e produção de Populus spp., no Chile (sistema Salica). Curitiba, 2003. 190 f. Tese (Doutorado em Manejo Florestal)Universidade Federal de Paraná.

BORDERS, B., SOUTER, R., BAILEY, R. e WARE, K. Percentile -Based distributions characterize forest stand table. Forest Sci., v.33, n.2, p. 570-576. 1987.
BURK, T.; NEWBERRY, J. A simple algorithm for moment-based recovery of Weibull distribution Parameters. Forest. Sci. v. 30, n. 2. p. $329-332.1984$.

CAO, Q.; BURKHART, H. A segmented distribution approach for modeling diameter frequency data. Forest Sci. V. 30, n. 1. p. 129 $-137.1984$

CAMPOS, J.C.C.; TURNBULL, K. Um sistema para estimar a produção por classe de diâmetro e sua aplicação na interpretação do efeito de desbaste. Revista Árvore, Universidade Federal Viçosa, v.5, n.1, p.1-16, 1981.

EISFELD, R.L. Modelagem do crescimento e da produção de Pinus taeda L. por meio de função probabilística e processo de difusão. Curitiba, 2004. 67f. Dissertação (Mestrado em manejo Florestal). Universidade Federal do Paraná.

FINGER, C.A.G. Distribuição de diâmetros em Acácia negra Acacia mearnsii de Wild, em diferentes povoamentos $\mathrm{e}$ idades. Curitiba, 1982, 129 f. Dissertação (Mestrado Manejo Florestal). Universidade Federal do Paraná,

GADOW, K.V. Fitting distributions in Pinus patula stands. South African Forestry Journal. Pretoria, n.126, p. 20 - 29, 1983.

GIBBONS, J. G.; CHAKRABORTI, S. Nonparametric Statistical Inference. Third Edition, Revised and Expanded. Editorial DEKKER, vol 131. New York, 1992. 544 p.

GLADE, J. E. Prognose de volume por classes diamétricas para Eucalyptus grandis Hill Ex-Maiden. Curitiba, Brasil, 1986. 94 f. Dissertação (Mestrado em Manejo Florestal). Universidade Federal de Paraná.

KANGAS, A.; MALTAMO, M. Calibrating predicted diameter distribution with additional information. Forest Sci. v. 46, n. 3. p. $390-$ 396. 2000

LEBART, L.; MORINEAU, A.; FÉNELON, J. D. Tratamiento Estadístico de datos, métodos y programas. Barcelona - México: Marcombo Boixareau Editores, 1985. 520 p. 
MAESTRI, R. Estimativa de produção presente futura de volume de madeira e peso de casca para povoamentos e acácia negra (Acacia Mearnsii de Wild). Curitiba, Brasil, 1992. 102 p. Dissertação (Mestrado em Manejo Florestal). Universidade Federal de Paraná.

MAESTRI, R. Modelo de crescimento e produção para povoamentos clonais de Eucalyptus grandis considerando variáveis ambientais. Curitiba, Brasil, 2003. $143 \mathrm{f}$. Tese (Doutorado em Manejo Florestal)- Pósgraduação em Engenharia Florestal, Universidade Federal de Paraná.

MALTAMO, M.; KANGAS, A.; UUTERRA, J.; TORNIAINEN, T. SARAMÄKI, J. Comparison of percentile based prediction an the Weibull distribution in describing the diameter distribuiton of heterogeneus Scots pine stands. Forest Ecology and Managment. v 133, p. $263-274.2000$

NEPAL, S.K. e SOMERS, G.L. A generalized approach to stand table projection. Forest Sci., v.38, n.1, p.120-1 33. 1992.
SCOLFORO, J. R. Sistema integrado para predição e análise presente e futura do crescimento e produção, com otimização de remuneração de capitais, para Pinus caribaea var. Hondurensis. Curitiba, 1990. 290 f. Tese (Doutorado em Ciências Florestais)- Pós-Graduação em Engenharia Florestal. Universidade Federal de Paraná.

SCOLFORO, J. R. Modelagem do crescimento e da produção de florestas plantadas e nativas. Lavras: UFLA/FAEPE. 1998. $441 \mathrm{p}$.

SILVA, J.A.A.; BAILEY, R.L. Predicting diameter distribution in fertilized slash pine plantations with the Weibull distribution. Acta Forestalia Brasiliensis, Curitiba, v.2, n.1, p.46-62, 1987.

THIERSCH, A. Eficiência das distribuições diamétricas para prognose da produção de Eucalyptus camaldulensis. Lavras, 1997. 155 f. Dissertação (Mestrado em Engenharia Florestal)- Universidade Federal Lavras. 